\title{
NK cell activating receptor ligand expression in lymphangioleiomyomatosis is associated with lung function decline
}

\author{
Andrew R. Osterburg, ${ }^{1}$ Rebecca L. Nelson, ${ }^{1}$ Benyamin Z. Yaniv, ${ }^{1}$ Rachel Foot, ${ }^{1}$ Walter R.F. Donica, \\ Madison A. Nashu, ${ }^{1}$ Huan Liu, ${ }^{1}$ Kathryn A. Wikenheiser-Brokamp, ${ }^{2,3}$ Joel Moss, ${ }^{4}$ Nishant Gupta, ${ }^{1}$ \\ Francis X. McCormack, ${ }^{1}$ and Michael T. Borchers ${ }^{1}$ \\ 'Department of Internal Medicine, Division of Pulmonary, Critical Care and Sleep Medicine, University of Cincinnati \\ College of Medicine, Cincinnati, Ohio, USA. '2Department of Pathology and Laboratory Medicine, University of Cincinnati, \\ Cincinnati, Ohio, USA. ${ }^{3}$ Division of Pathology \& Laboratory Medicine and Perinatal Institute, Division of Pulmonary \\ Biology, Cincinnati Children's Hospital Medical Center, Cincinnati, Ohio, USA. ${ }^{4}$ Cardiovascular and Pulmonary Branch, \\ National Heart, Lung, and Blood Institute, NIH, Bethesda, Maryland, USA.
}

Lymphangioleiomyomatosis (LAM) is a rare lung disease of women that leads to progressive cyst formation and accelerated loss of pulmonary function. Neoplastic smooth muscle cells from an unknown source metastasize to the lung and drive destructive remodeling. Given the role of NK cells in immune surveillance, we postulated that NK cell activating receptors and their cognate ligands are involved in LAM pathogenesis. We found that ligands for the NKC2D activating receptor UL-16 binding protein 2 (ULBP2) and ULBP3 are localized in cystic LAM lesions and pulmonary nodules. We found elevated soluble serum ULBP2 (mean $=575 \mathrm{pg} / \mathrm{ml} \pm 142$ ) in 50 of 100 subjects and ULBP3 in 30 of 100 (mean $=8,300 \mathrm{pg} / \mathrm{ml} \pm 1,515$ ) subjects. LAM patients had fewer circulating NKC2D+ NK cells and decreased NKG2D surface expression. Lung function decline was associated with soluble NKC2D ligand (sNKC2DL) detection. The greatest rate of decline forced expiratory volume in 1 second ( $\mathrm{FEV}_{1},-124 \pm 30 \mathrm{ml} /$ year) in the 48 months after enrollment (NHLBI LAM Registry) occurred in patients expressing both ULBP2 and ULBP3, whereas patients with undetectable sNKG2DL levels had the lowest rate of FEV decline $(-32.7 \pm 10 \mathrm{ml} /$ year $)$. These data suggest a role for NK cells, sNKG2DL, and the innate immune system in LAM pathogenesis.

Conflict of interest: The authors have declared that no conflict of interest exists.

Submitted: March 10, 2016 Accepted: August 30, 2016 Published: October 6, 2016

Reference information: JCI Insight. 2016;1(16):e87270. doi:10.1172/jci.insight.87270.

\section{Introduction}

Lymphangioleiomyomatosis (LAM) is a rare, low-grade, metastasizing neoplasm that results in progressive cystic lung disease and respiratory failure. Symptomatic LAM occurs almost exclusively in women. LAM occurs in patients with tuberous sclerosis complex (TSC-LAM) who have germ line mutations in TSC genes and in patients who do not have TSC (termed sporadic LAM [S-LAM]) (1) but acquired somatic mutations within LAM lesions. Although the source of LAM cells is unknown, the leading hypothesis for LAM pathogenesis is that inactivating mutations in the TSC genes (TSC1 or TSC2) occur within peripheral tissues, resulting in mTOR activation, loss of growth control, and enhanced capacity for migration and infiltration. These LAM cells then spread via the blood and lymphatics and selectively populate the lung, causing destructive remodeling (2) by mechanisms that remain unclear.

The higher rates of various cancers in immunocompromised humans and mice attests to the importance of adaptive and innate immune surveillance in the protection from neoplasia (3). However, tumor cells can also escape immune detection in immunocompetent hosts by acquiring mutations or other alterations. The NKG2D (killer cell lectin like receptor K1 [KLRK1], CD314) activating receptor expressed on NK cells and other cytotoxic lymphocytes recognizes stressed, infected, or transformed cells that express NKG2D ligands (NKG2DLs) and targets them for destruction (4) by triggering cytotoxic programs (5). Engagement of NKG2DLs by the NKG2D receptor induces target cell lysis (5-8) by activation of the perforin/granzyme cell death pathway $(9,10)$ and elaboration of proinflammatory cytokines $(7,11)$ and chemokines $(7,12,13)$.

NKG2DL expression is restricted or absent in normal tissues but is induced in response to various stresses and pathological conditions. Multiple families of structurally distinct NKG2DLs have been identi- 

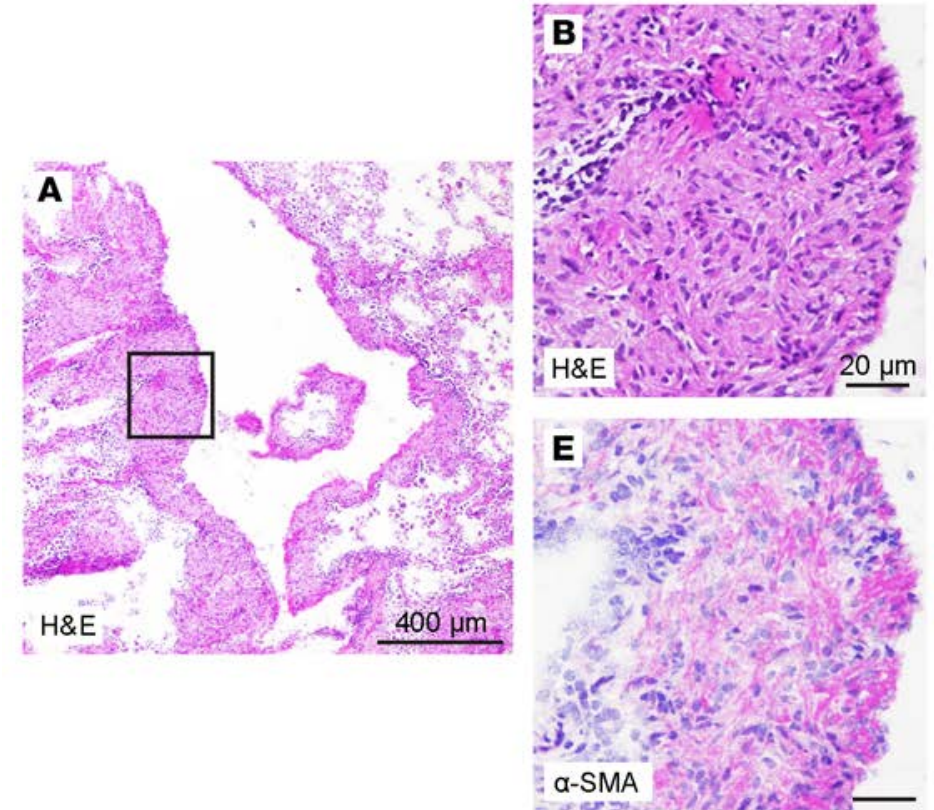
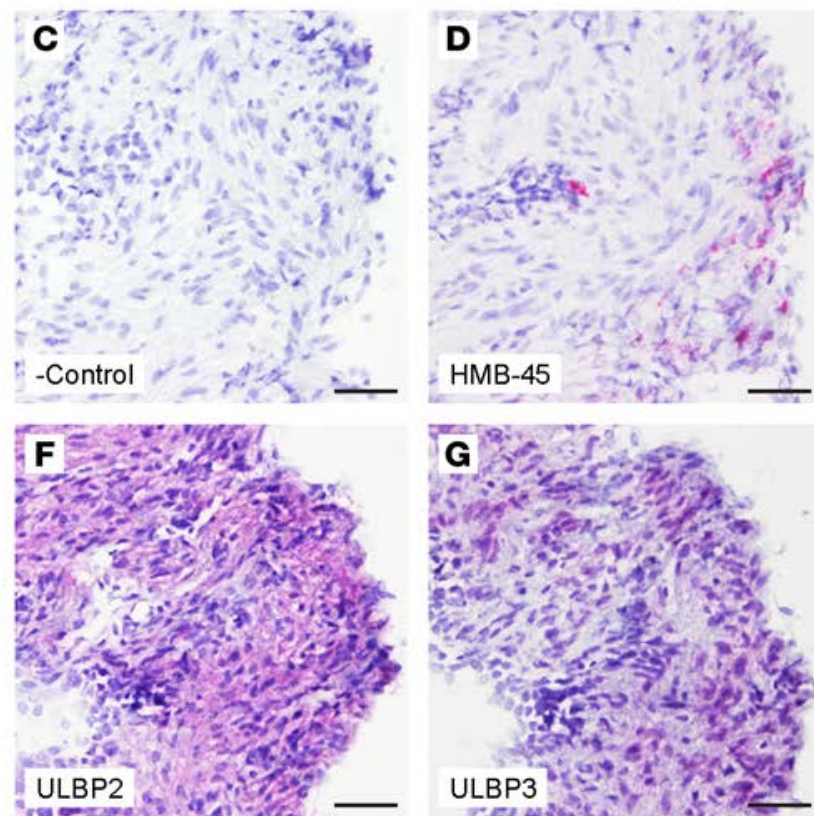

Figure 1. NKG2D ligands are expressed in lymphangioleiomyomatosis (LAM) cells in lung biopsy specimens from LAM patients. Serial diagnostic lung tissue sections from LAM patients were stained for the LAM cell markers HMB-45 and $\alpha$-SMA, as well as for NKG2D ligands ULBP2 and ULBP3. (A) Representative cystic LAM lesion with LAM cell aggregates adjacent to the cyst (boxed area) identified by routine H\&E staining. Scale bar: $400 \mu \mathrm{m}$. (B) Higher magnification image of the boxed region in $\mathbf{A}$ and images of serial sections of the same region IHC stained for (D) HMB-45, (E) $\alpha-S M A,(\mathbf{F})$ ULBP2, and (C) ULBP3, along with (C) a representative negative control. LAM cells comprising the cellular aggregate adjacent to the LAM cyst show characteristic strong focal staining (red) for HMB-45, diffuse staining for $\alpha$-SMA, and positive staining for the NKC2D ligands ULBP2 and ULBP3. No staining was observed in multiple negative control sections for all antibodies, as depicted by the representative negative control image shown. Scale bars: $20 \mu \mathrm{m}$ (B-G). Supplemental Figure 1 shows representative staining in diagnostic lung biopsies from 6 additional LAM patients.

fied in humans, including the MHC class I chain-related (MIC) molecules, MICA and MICB (5), and the UL-16 binding proteins, ULBP1, $-2,-3,-4,-5$, and $-6(7,14,15)$. Although expression of NKG2DLs has been described in many human cancers - including melanoma, leukemia, and carcinomas of the prostate, breast, lung, and colon (16) - the role of NKG2DLs in LAM and other low-grade mesenchymal neoplasms has not been explored.

Shedding of NKG2DLs from tumor cells is a well characterized "decoy" mechanism by which neoplastic cells escape immune detection. Soluble MICA and ULBPs are detectable in the serum of patients with various tumors (17) and have been shown to interfere with the recognition of target cells by the NKG2D receptor. Circulating levels of soluble NKG2DLs (sNKG2DL) are useful diagnostic and prognostic biomarkers in certain cancers, as they have been shown to discriminate between patients with pancreatic adenocarcinoma and healthy controls (18) and have been used to predict the risk of disease progression in melanoma patients (19).

Here, we examined NKG2DL expression in LAM lesions, soluble serum NKG2DLs, circulating NK cell phenotypes, and the association of sNKG2DLs and disease progression in LAM. We found that the smooth muscle actin-expressing (SMA-expressing) LAM cells within nodules and cysts express multiple NKG2DLs and that sNKG2DLs were present in LAM serum. Furthermore, we show that NK cells are phenotypically altered in LAM patients, as measured by decreased NKG2D receptor expression on NK cells. Finally, we show that the presence of sNKG2DLs is associated with a greater decline in lung function over time in LAM patients. These data suggest that LAM-dependent alterations in NKG2DL and the NK milieu are biomarkers of lung destruction and progression, and implicate NK cells in the pathophysiology of LAM.

\section{Results}

NKG2DL expression is restricted to LAM lesions in human lung biopsy specimens. To examine NKG2DL expression in LAM, we obtained frozen specimens obtained from the 5-year National Heart Lung and Blood Institute (NHLBI) LAM Registry and maintained by the National Disease Research Interchange (NDRI) tissue repository. These samples were stained for NKG2DLs (ULBP2 and ULBP3). Diagnostic features 
Table 1. National Heart Lung and Blood Institute (NHLBI) patient demographics

\begin{tabular}{|c|c|c|c|c|}
\hline & Neither & ULBP2 & ULBP3 & Both \\
\hline$n$ & 37 & 33 & 12 & 18 \\
\hline $\mathrm{FEV}_{1}$ & $1.9 \pm 0.7$ & $1.9 \pm 0.9$ & $2.6 \pm 0.7$ & $2.4 \pm 0.9$ \\
\hline FVC & $2.99 \pm 0.8$ & $3.08 \pm 0.8$ & $3.38 \pm 0.7$ & $3.33 \pm 1.1$ \\
\hline TLC & $4.79 \pm 1.2$ & $4.95 \pm 1.4$ & $4.83 \pm 0.7$ & $4.89 \pm 1.5$ \\
\hline \multicolumn{5}{|c|}{ FEV $_{1} /$ FVC $>70 \%$} \\
\hline$n$ & 11 & 10 & 9 & 9 \\
\hline Age & $44.0 \pm 7.10$ & $44.1 \pm 10.6$ & $38.1 \pm 9.42$ & $40.7 \pm 8.25$ \\
\hline $\mathrm{FEV}_{1}$ & $2.39 \pm 0.40$ & $2.50 \pm 0.59$ & $2.88 \pm 0.48$ & $2.79 \pm 0.66$ \\
\hline FVC & $3.06 \pm 0.42$ & $3.17 \pm 0.63$ & $3.52 \pm 0.71$ & $3.52 \pm 0.85$ \\
\hline
\end{tabular}

$\mathrm{FEV}_{1}$, forced expiratory volume in one second; FVC, forced vital capacity; TLC, total lung capacity; DLCO, diffusing capacity. Data shows mean \pm SD.

of LAM were confirmed in the tissue sections by histologic analysis, as well as by LAM cell immunophenotype, including positive staining for human melanoma black-45 (HMB-45) and $\alpha$-SMA. ULBP3 and/ or ULBP2 expression was detected in LAM cells within all 7 tested lung biopsy samples representing 7 distinct LAM patients (Figure 1 and Supplemental Figure 1; supplemental material available online with this article; doi:10.1172/jci.insight.87270DS1). We further examined localization of NKG2DL expression with expression of the LAM cell markers $\alpha$-SMA and HMB-45. Substantial coexpression of the NKG2DLs ULBP2 and ULBP3 with $\alpha$-SMA was detected in LAM cells (Figure 2). In addition, HMB-45 was expressed in a subpopulation of cells within the LAM lesions also expressing NKG2DLs and $\alpha$-SMA (Figure 3). NKG2DL expression was not detected in regions of the lung uninvolved by LAM lesions (Supplemental Figure 2), providing evidence that NKG2DL expression is associated with LAM pathology.

Increased sNKG2DLs in the serum of LAM patients. NKG2DL expression in lung tissue may serve as a source of sNKG2DL, which is associated with alterations in NK cell phenotype and disease progression in other neoplastic diseases. Therefore, we measured the serum levels of MICA, ULBP2, and ULBP3 in samples from LAM patients who participated in the NHLBI LAM Registry, and we evaluated correlations with their longitudinal spirometry and clinical data. Demographic data of LAM patients at enrollment is shown in Table 1 . We found that patients had detectable serum levels of either ULBP2, $(n=33)$, ULBP3 $(n=12)$, or both $(\mathrm{ULBP} 2 / 3)(n=18)$ ligands (Figure 4 , and Table 1$)$. In contrast, significant levels of soluble MICA were not found (data not shown). The serum levels of 2 additional known immune checkpoint modulators, cytotoxic T-lymphocyte-associated protein 4 (CTLA-4) and programmed death-ligand 1 (PD-L1), were also low or undetectable (data not shown). Additionally, serum ULBP2/ULBP3 levels and baseline to endpoint change in forced expiratory volume in 1 second $\left(\mathrm{FEV}_{1}\right)$ were not significantly correlated (ULBP2 at 30 and 48 months, $\mathrm{r}=-0.029$ and -0.104 , respectively; ULBP3 at 30 and 48 months, $r=-0.264$ and -0.212 , respectively). In addition, there were no between-group differences in vascular endothelial growth factor D (VEGF-D) levels (data not shown) among NKG2DL expression cohorts (i.e., ULBP2, ULBP3, or ULBP2/3 present or absent; all patients at enrollment grouped by sNKG2DL versus VEGF-D serum levels $P=0.608$; patients included at 48 months with enrollment $\mathrm{FEV}_{1} /$ forced vital capacity $[\mathrm{FVC}]>0.7, P=0.187$ ). There was also no significant correlation between VEGF-D serum levels and sNKG2DL levels (Spearman correlation coefficients between ULBP2 and ULBP3 [ $\rho=-0.059]$, ULBP2/VEGF-D [ $\rho=-0.202]$, and ULBP3/VEGF-D ( $\rho=-0.148)$ ]. Furthermore, because menopausal status is known to have a major impact on disease progression in LAM, we also examined the effect of menopausal state on NKG2DL expression. There was no correlation between NKG2DL group assignment (Neither, ULBP2, ULBP3, $\mathrm{ULBP} 2 / 3)$ and menopausal status $\left(\chi^{2}, P=0.28\right)$. 

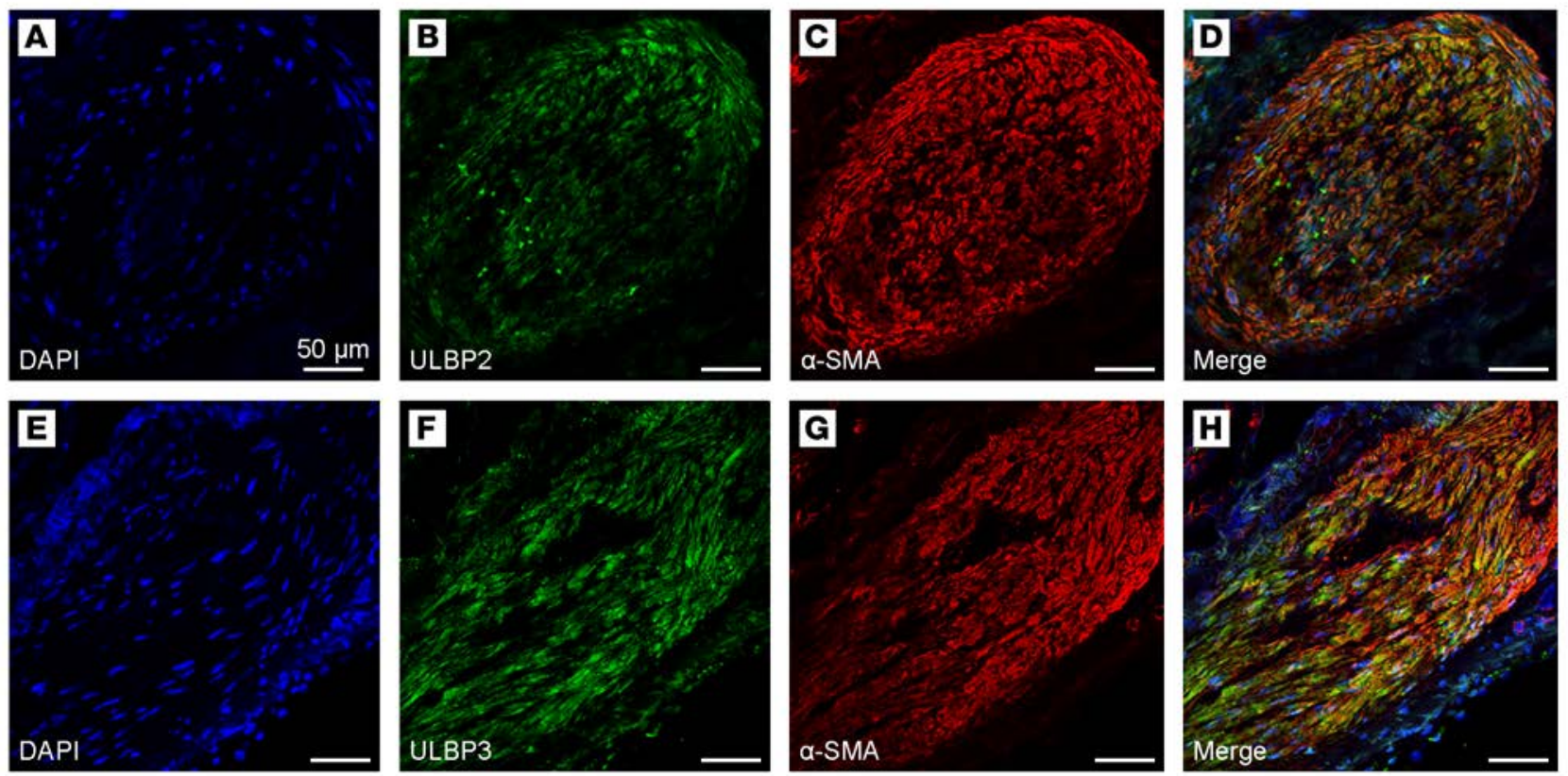

Figure 2. NKG2D ligand an $\alpha$-SMA localization in lymphangioleiomyomatosis (LAM) cells in lung biopsy specimens from LAM patients. (A-D and E-H) Immunofluorescent images show nodular LAM lesions from 2 distinct LAM patients. LAM lung tissue sections were stained for DAPI (A and E), the NKC2D ligands (B, green) ULBP2 and (F, green) ULBP3, as well as for the LAM cell marker (C, G, red) $\alpha$-SMA. Confocal microscopy images show immunofluorescent localization of the NKG2D ligands (D) ULBP2 and (H) ULBP3 with $\alpha$-SMA within the LAM cells comprising the nodular LAM lesions. Scale bars: $50 \mu \mathrm{m}$. The images are representative of results obtained by immunofluorescent staining of diagnostic lung biopsies from 9 LAM patients.

NK cells exhibit reduced expression of NKG2D in LAM patients. Due to the presence of elevated levels of sNKG2DLs in the sera of some LAM patients, we examined the phenotype of NK cells from the peripheral blood of healthy controls and 7 LAM patients recruited from the University of Cincinnati LAM Clinic. Figure 5A shows a representative scatterplot of NKG2D and NKG2C (killer cell lectin like receptor C2 [KLRC1], CD159c) activating receptors of a healthy control subject and a LAM patient. Changes in the relative abundance of NK subsets was not due to alterations in overall levels of either cytokine-producing $\mathrm{CD} 56^{\text {bight }} \mathrm{CD} 16^{-}$or cytotoxic $\mathrm{CD} 56^{\mathrm{dim}} \mathrm{CD} 16^{++} \mathrm{NK}$ cell subpopulations (Figure $5 \mathrm{~B}$ ). NKG2C levels trended toward reduced surface expression but were not statistically significant (Figure 5C). LAM patients had fewer NKG2D (\% positive) cells, as well as fewer NKG2D cell surface receptors (median fluorescent intensity [MFI]) compared with healthy controls. Figure 5D demonstrates reduced surface expression of NKG2D on both $\mathrm{CD} 56^{\text {bright }} \mathrm{CD} 16^{-}(P<0.024)$ and $\mathrm{CD} 56^{\mathrm{dim}} \mathrm{CD} 16^{++}(P<0.002) \mathrm{NK}$ cells of LAM patients compared with control subjects. Consistent with the reduced expression of NKG2DL on a per-cell basis, there were also fewer cells positive for NKG2D in $\mathrm{CD} 56^{\text {bright }} \mathrm{CD} 16^{-}$and $\mathrm{CD} 56^{\mathrm{dim}} \mathrm{CD} 16^{++}$populations from LAM patients compared with healthy controls, $(P<0.032$ and $P<0.06$, respectively) (Figure 5E). Of the LAM patients tested, 0 of 7 were positive for sULBP2 and 3 of 7 were positive for sULBP3, whereas none of the controls had detectable quantities of ULBP2 or ULBP3 (data not shown). Of these patients, 4 of 7 were receiving either sirolimus or everolimus (Supplemental Table 1). Taken together, these data are consistent with reduced NKG2D expression on circulating NK cells that could implicate altered NK cell phenotypes in the peripheral blood in the pathogenesis of LAM.

Expression of $S N K G 2 D L S$ is associated with lung function decline in patients with LAM. Next, we examined the relationship between the presence and absence of sNKG2DL in serum and disease progression as defined as the rate of decline in $\mathrm{FEV}_{1}$ over time. Pulmonary function measurements were obtained during annual or semiannual visits in the NHLBI LAM Registry study (20). Serum obtained on the first visit was used to determine the association between baseline sNKG2DL expression and change in FEV ${ }_{1}$ over 30 or 48 months after enrollment (Figure 6). To limit the impact of missing data, intraindividual $\mathrm{FEV}_{1}$ data from the 24- to 36-month visits were averaged and denoted as the 30-month $\mathrm{FEV}_{1}$, and $\mathrm{FEV}_{1}$ data from the 42-54 month visits were averaged and represented as the $48-\mathrm{month}_{\mathrm{FEV}}$. We also separately examined the subgroup of 

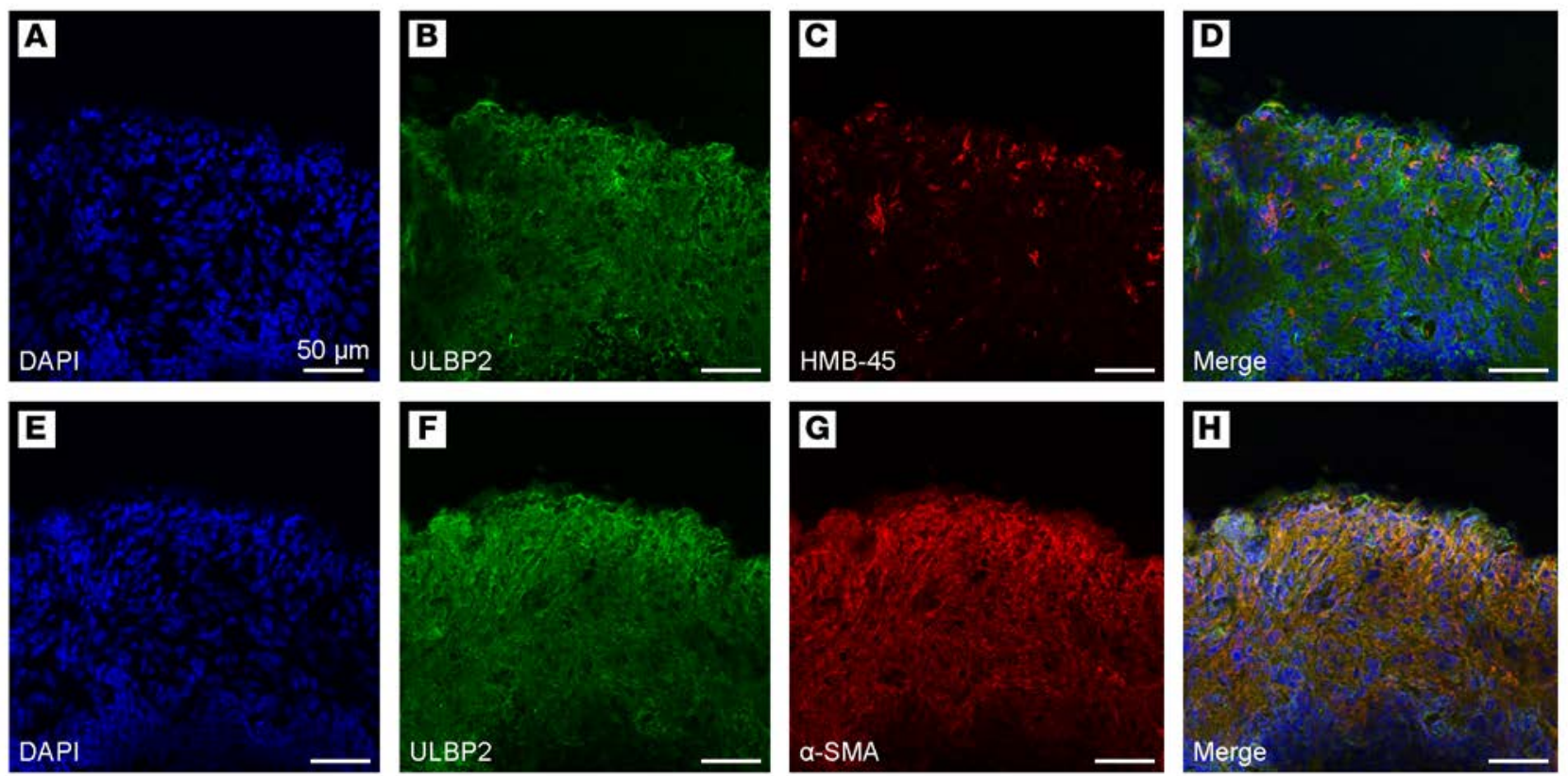

Figure 3. HMB-45 expression is present in lymphangioleiomyomatosis (LAM) cell aggregates coexpressing NKG2D ligands and $\alpha$-SMA in lung biopsy specimens from LAM patients. (A-D and E-H) Immunofluorescent images of serial sections of LAM cell aggregates surrounding a LAM cyst stained for (A and E) DAPI, (B and F) ULBP2, (C) HMB-45, and (C) $\alpha$-SMA. Confocal microscopy images show HMB-45 expression within a subpopulation of LAM cells comprising the LAM cell aggregate coexpressing the NKG2D ligand ULBP2 and $\alpha-S M A$ ( $\mathbf{D}$ and $\mathbf{H}$ ). Scale bars: $50 \mu \mathrm{m}$. The images are representative of results obtained by immunofluorescent staining of diagnostic lung biopsies from 7 LAM patients.

patients with normal lung function, defined as $\mathrm{FEV}_{1} / \mathrm{FVC}>70 \%$, because biomarkers that predict disease progression in this category could have a pivotal impact on treatment decisions. Figure $6 \mathrm{~A}$ shows the loss in $\mathrm{FEV}_{1}$ at 30 and 48 months compared with $\mathrm{FEV}_{1}$ at enrollment. Patients with baseline elevations in serum of both sNKG2DLs (ULBP2/3) had a greater decline in lung function over 48 months compared with patients who had baseline levels of sNKG2DL below the limit of detection in their serum (48 months, Neither, -103 $\pm 70 \mathrm{ml}$ vs. 48 months, Both, $-497 \pm 120 \mathrm{ml}$, \pm SEM, $P<0.002)$. Moreover, the presence of both sNKG2DL (ULBP2/3) was associated with a significantly greater $\mathrm{FEV}_{1}$ loss at 30 months compared with patients who had no detectable sNKG2D in serum (30 months, ULBP2/3, $-350 \pm 110 \mathrm{ml}$ vs. 30 months, Neither, $-80 \pm$

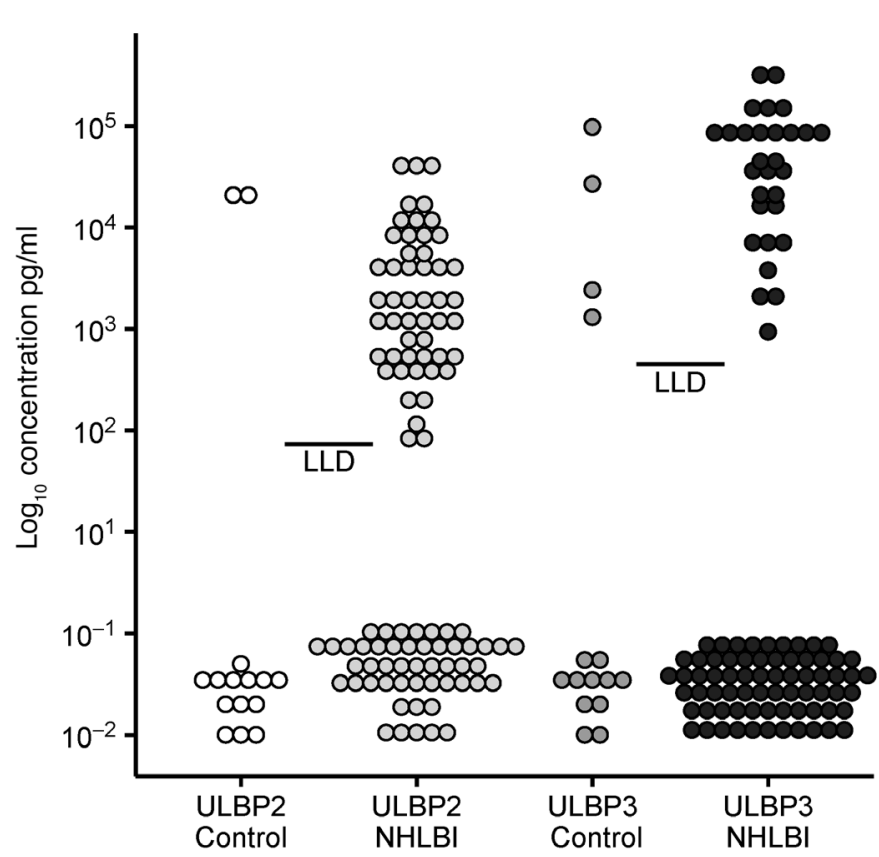

$50 \mathrm{ml}, \pm \mathrm{SEM}, P<0.021)$. Patients who were positive for serum ULBP3 at enrollment also suffered a greater baseline to 48-month loss of $\mathrm{FEV}_{1}$ compared with those who had undetectable baseline ULBP3 levels (48 months, ULBP3, $-379 \pm 50 \mathrm{ml}$ vs. 48 months, Neither, $-103 \pm 70 \mathrm{ml}, \pm \mathrm{SEM}, P<0.037)$. These findings suggest that the presence of multiple sNKG2DLs may be a useful predictor of lung function decline in LAM.

\section{Discussion}

LAM results from inherited or acquired loss-of-function mutations in the TSC genes (TSC1/2). Transformed smooth musclelike cells arise from an unknown source, metastasize via blood and lymph (21), infiltrate the lung (1), and proliferate, forming nodules

Figure 4. Soluble NKG2D Ligands in National Heart Lung and Blood Institute lymphangioleiomyomatosis (NHLBI LAM) Registry patients. Soluble NKG2D ligands were measured in serum of NHLBI LAM Registry patients and healthy volunteers (control). Bar represents the lower limit of detection (LLD) for each analyte. $n=15$ for controls, $n=100$ for NHLBI samples. 
A

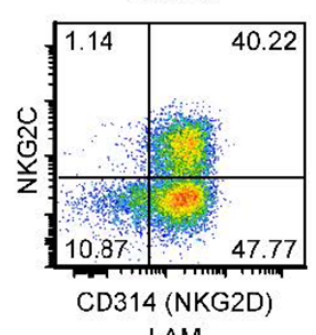

LAM

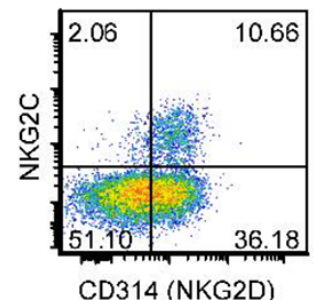

B

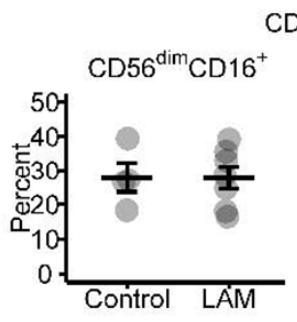

D

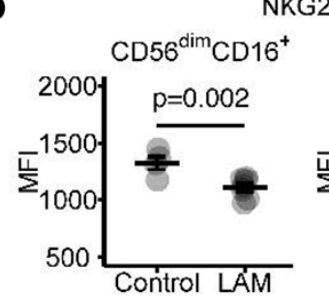

CD3-

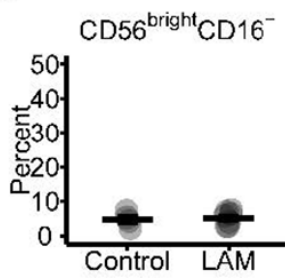

KG2D

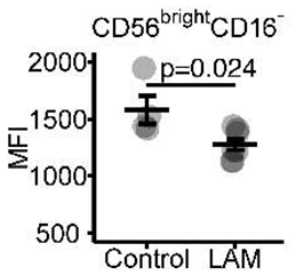

C

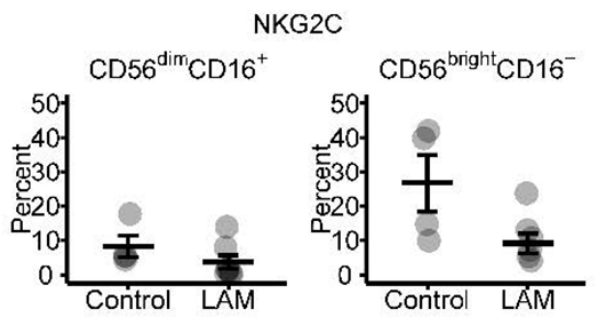

E

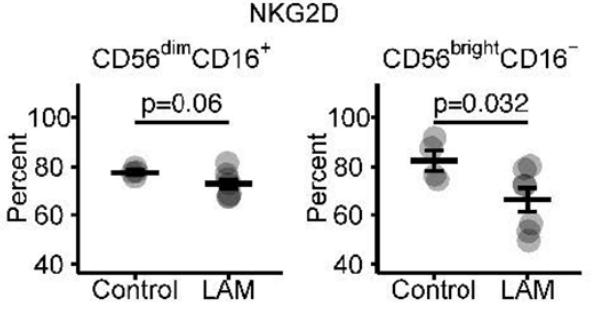

Figure 5. NKG2D expression on NK cells in peripheral blood. (A) Scatterplot representative of control and lymphangioleiomyomatosis (LAM) patient. (B) Plots indicating percent positive for $\mathrm{CD}^{-}{ }^{-} \mathrm{CD} 56^{\text {bright }} \mathrm{CD} 16^{-}$and $\mathrm{CD}^{-} \mathrm{CD}^{\mathrm{dim}} \mathrm{CD}^{\mathrm{di}} 6^{++}$NK cells. Sample size for B-D, controls, $n=4$; LAM patients, $n=7$. (C)

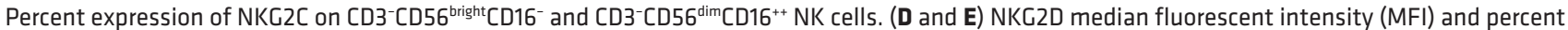
positive on $\mathrm{CD}^{-} \mathrm{CD}_{5} 6^{\text {bright }} \mathrm{CD} 16^{-}$and $\mathrm{CD}^{-} \mathrm{CD}^{-\mathrm{dim}} \mathrm{CD}^{-{ }^{++}} \mathrm{NK}$ cells. Statistical significance indicated by $P$ value of LAM group vs. controls. An unpaired 2-tailed Student's $t$ test was used to determine statistical significance. Bar represents mean \pm SEM.

and cysts. Destructive remodeling leads to loss of lung parenchyma and capillary beds, ultimately leading to disease manifestations of dyspnea, pneumothorax, chylous effusions, and respiratory failure. The strategies that LAM cells employ to evade immune detection and to drive destructive remodeling are not clear. Here, we report that NKG2DLs are expressed in LAM nodules and cysts, LAM patients have circulating NK cells with altered phenotypes in peripheral blood, and sNKG2DLs are present in the serum and are associated with accelerated lung function decline.

The surface expression of NKG2DL on transformed or stressed cells results in engagement of NKG2D on NK or CD8 cytotoxic T cells followed by target cell lysis. Immune surveillance by NKG2D-NKGD2L interactions plays a critical role in suppressing tumor initiation in mice and humans (22). Numerous reports demonstrate sNKG2DL upregulation and concomitant inhibition of NK cell tumor suppression in various cancers and virally infected tissues $(19,23-27)$. Transcriptional, and pre-, and posttranslational mechanisms are involved in regulating the expression of NKG2DL. Each NKG2DL (e.g., MICA, ULBP2, ULBP3, etc.), however, appears to be regulated independently of the others. NKG2DLs ULBP2 and/or ULBP3 were present in all nodules and cystic lung lesions tested. NKG2DL expression within LAM tissue is consistent with the classification of LAM as a neoplasm $(2,28)$. The role of $T S C 1 / 2$ mutations in the expression of NKG2DL is unknown, but intersections between signaling pathways that regulate NKG2DL cell surface expression and PI3K/Akt/mTOR signaling known to play a role in LAM pathogenesis have been described in the literature. For example, EGFR signaling is enhanced in cells isolated from LAM lesions $(29,30)$, and surface expression of NKG2DL is upregulated upon activation of EGFR, which signals through mTOR and MEK/ERK pathways (31-33). Moreover, inhibition of the MEK/ERK pathway decreases cell surface expression of ULBP2 and ULBP3 (34), and PI3K/AKT/mTOR-dependent signaling has been shown to regulate NKG2D expression (35). Taken together, these data provide evidence for marked crosstalk between the mTOR pathway and signaling cascades that regulate NKG2DLs, suggesting that TSC mutations may directly modulate NGK2DL expression.

NKG2DL shedding may play a role in LAM pathogenesis. The release of sNKG2DL from transformed cells reduces the surface density of NKG2DLs and the potential for NKG2D-mediated lysis of target cells. In addition, elevated NKG2DL in the serum may bind to cell surface NKG2D and promote the internalization of the ligand-receptor pair, reducing NK cell effector functions $(16,17)$. Proposed mechanisms of NKG2DL shedding from tumor cells or stressed cells include proteolytic cleavage of cell-surface ligand by matrix metalloproteases (MMP) (36), a disintegrin and metalloproteinase (ADAM) (24), or phospholipase C (37) or release of NKG2DLs in an exosome-dependent manner $(38,39)$. Circumstantial evidence for at least 2 of these shedding mechanisms has been described in LAM. Matsui et al. demonstrated elevated 
A

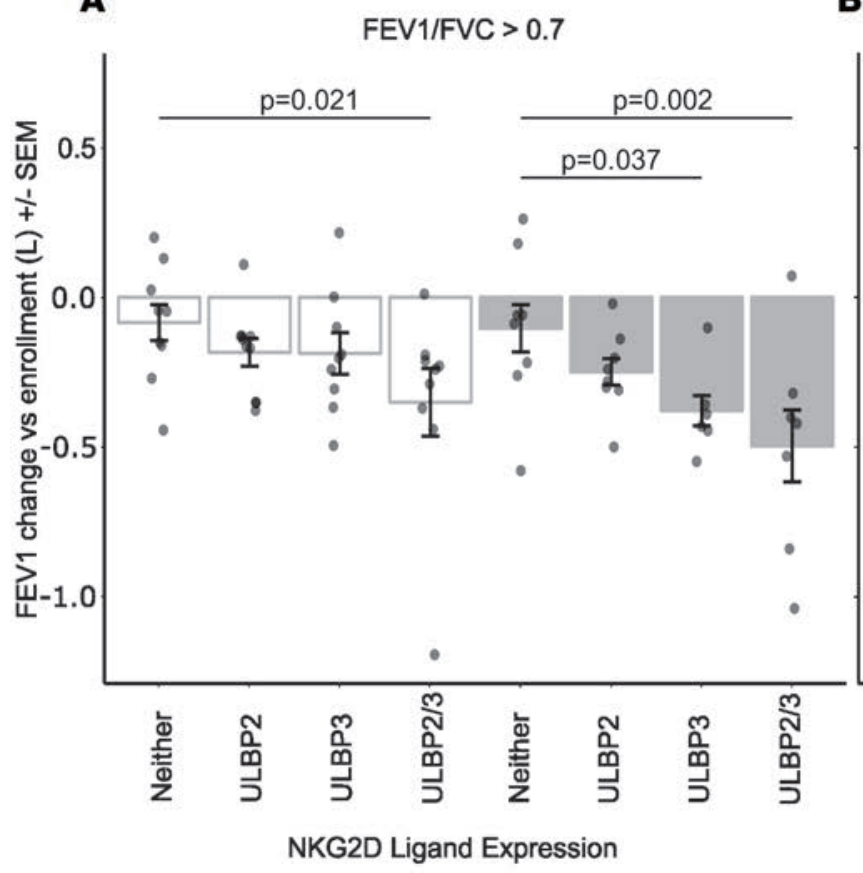

B

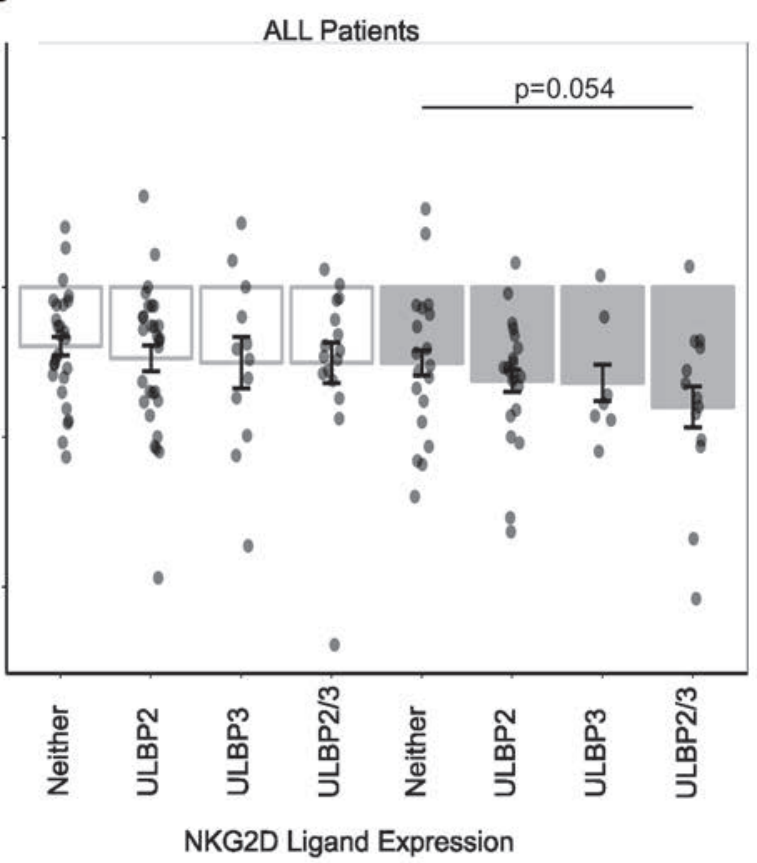

Figure 6. Decline in forced expiratory volume in 1 second (FEV) based on presence or absence of serum sNIKG2D ligands. Patients were grouped by the presence or absence of soluble NKC2D ligands (Neither, ULBP2, ULBP3, or ULBP2/3). Mean change from baseline to 30 months (white filled) or 48 months (gray filled) are shown. (A) Data shown is for individuals with $\mathrm{FEV}_{1} /$ forced vital capacity (FVC) $>0.70$ at enrollment. $n=7-9 /$ group (B) Data shown is for all patients $n=7-29$ /group. Statistical significance between groups was determined using a 2-way ANOVA, with specific post-hoc contrasts for group comparisons. Dots represent patients. "Data are presented as bar plots with the height of the bar representing the mean and error bars \pm SEM.

MMP2 activation in pathologic lesions of LAM patients (40), and Edwards et al. demonstrated increased transcripts for MMPs, ADAMs, and tissue inhibitor of metalloproteinases (TIMPs) within mast cells isolated from LAM biopsies (41). Despite the remarkably consistent detection of NKG2DL in LAM tissue specimens by IHC, there is considerable variation in the presence and quantity of sNKG2DL in serum of LAM patients, perhaps explained in part by differences in the proteolytic milieu in LAM lesions.

Few studies have examined NK cells in the context of LAM pathogenesis. Our studies reveal that NK cells in LAM patients demonstrate both reduced expression of NKG2D and a trend toward reduced NKG2C expression on peripheral blood NK cells. Our data indicate that there are LAM-associated changes in NK cell populations in peripheral blood and suggest that altered NK function may also play a role in LAM pathogenesis, including reduced tumor surveillance and altered cytotoxicity of NK cells. It is well established that upregulation of sNKG2DLs results in decreased NKG2D expression and functional alterations in NK cells (23). However, since only a portion of the LAM patients had sNKG2DL, the presence of sNKG2DLs alone does not explain the consistent reduction in NKG2D expression observed in NK cell populations of all the LAM patients examined. Additionally, these studies are limited by the small sample size and absence of longitudinal lung function data in this cohort. Reduced NKG2D expression has been reported under several conditions. Altered NK ligand expression and NK cell function in LAM may be related to the hypoxemia experienced by LAM patients and the hypoxic environment within LAM nodules $(42,43)$. Hypoxic conditions are reported to impair NK cell antiviral activity (44), downregulate activating receptors (45), and impair cytotoxicity against myeloma (46). Alternatively, soluble factors, such as cytokines, chemokines, or growth factors, present in LAM patients may modulate NKG2D expression. TGF- $\beta$ expression, which is known to be associated with downregulation of NKG2D in cancer $(47,48)$, is present in proliferative nodular LAM lesions (49) and may also influence receptor expression in LAM. Moreover, there may be direct effects of rapamycin/rapamycin analogs on NK cells, as mTOR is known to be an important regulator of $\mathrm{NK}$ cell effector function (50). Although systemic oxidative stress does not appear to be part of the pathophysiology of LAM (51), NKG2D surface expression can be reduced due to systemic oxidative stress, such as in end-stage renal disease (52). However, the exact mechanism for downregulation of NKG2D in peripheral blood NK cells in LAM patients remains unknown. 
LAM lesions in all lung biopsies we examined were positive for NKG2DL expression; however, the presence of sNKG2DLs was highly variable among patients. The explanation for this dissociation between expression in the tissue lesions and in the serum is not clear. MICA, and to a lesser extent ULBPS, are highly polymorphic genes with nucleotide variants found in coding and noncoding regions (53), and specific NKG2DL haplotypes may contribute to differential NKG2DL shedding and susceptibility to disease (54). For example, specific MICA haplotypes are associated with idiopathic pulmonary fibrosis (55). Specific ULBP NKG2DLs may also contribute to disease pathogenesis - for example, in alopecia areata (56). However, haplotype specific contributions of ULBPs to disease pathophysiology have yet to be elucidated (57). Heterogeneity in TSC1/2 mutations may also contribute to alterations in downstream signaling that result in differential upregulation of NKG2DL expression. The sNKG2DL data presented in the current study represent a single time point at enrollment into the NHLBI five-year LAM study. How these ligands change over the course of the disease progression - and how they may contribute to LAM pathophysiology - remains to be elucidated.

We found that the presence of both soluble ULBP2 and ULBP3 in serum is associated with longterm reduction in pulmonary function in patients with LAM. The finding that NKG2DLs are expressed in all LAM lesions in lung biopsies indicates that the release of these ligands from the tissue may play a role in LAM disease progression. There are no data describing the mechanisms by which LAM cells become implanted in the lung, proliferate, and culminate in cystic remodeling and loss of lung function. Our current data lead us to postulate that the proliferating LAM smooth muscle-like cells upregulate NKG2DL expression in the tissue of origin and are either eliminated or escape NK cell detection, become implanted into the lung, and further escape NK cell detection via the release of sNKG2DLs. We believe that release of sNKG2DLs is a critical event in disease progression, leading to reduced or occupied NKG2D receptors that permit the formation and growth of nodules that evolve to cystic lesions through unknown mechanisms. Further research is needed to elucidate the mechanisms for NKG2DL expression and shedding and for the modulation of NK cell function that contributes to disease pathogenesis in LAM patients. A more complete understanding of NK and sNKG2DL function in LAM pathogenesis could lead to development of NK-based interventional targets and perhaps inform treatment decisions in the future. Clinical trials that target NKG2DLs (58-60) in other neoplastic diseases are underway and may reveal strategies that could be successfully applied to LAM.

\section{Methods}

Human samples. Serum samples and longitudinal lung function data were obtained from the 5-year NHLBI LAM Registry (20) database and repository, curated by the NDRI (Philadelphia, Pennsylvania, USA). Samples were stored at $-80^{\circ} \mathrm{C}$ until analysis. Fresh blood from healthy female volunteers $(n=4$, ages: $26,38,42,48$; mean $[ \pm \mathrm{SD}]=38.5 \pm 9.2)$ and LAM patients $(n=7$, ages: $41,45,48,47,52,52,62$; mean $[ \pm \mathrm{SD}]=49.5 \pm 6.7)$ was obtained at the University of Cincinnati Pulmonary LAM Clinic after obtaining informed consent (IRB 2013-8157) (Supplemental Table 1). Blood was immediately processed to isolate peripheral blood mononuclear cells (PBMC) by Lymphoprep (Stemcell Technologies) density gradient centrifugation, and PBMC were cryopreserved in freezing media (50\% RPMI 1640, 40\% FBS, 10\% DMSO) using a Mr. Frosty Freezing Container (Thermo Fisher Scientific).

$I H C$. LAM lung biopsy sections $(n=9)$ obtained from NDRI were embedded in OCT medium and flash frozen in liquid nitrogen. Frozen sections $(7-\mu \mathrm{m}$ thickness) were prepared by the Pathology Research Core at Cincinnati Children's Hospital Medical Center and stained with H\&E for histopathological analysis or with antibodies specific to NKG2DL in order to determine the localization and abundance of ULBP2 and ULBP3. The following rabbit polyclonal primary antibodies obtained from Novus Biologicals were used: ULBP2 (catalog 27080002) and ULBP3 (catalog NBP2-31866). $\alpha$-SMA monoclonal antibody (clone 1A4) was obtained from Abcam. Melanoma (gp100) Ab-1 (clone HMB45) was purchased from Thermo Fisher Scientific. Frozen slides were equilibrated at room temperature (RT) prior to fixation for 5 minutes with $90 \% \mathrm{MeOH}$ at $-20^{\circ} \mathrm{C}$. After blocking of endogenous peroxidase and biotin, tissue sections were incubated in normal goat serum for 1 hour at RT. Slides were incubated with primary antibodies ULBP2 (1:2,000), ULBP3 (1:500), and HMB45 (1:150) overnight at $4^{\circ} \mathrm{C}$. The following day, slides were incubated with biotinylated goat anti-rabbit secondary antibody $(1: 1,000)$ for 2 hours at RT. Detection was performed using streptavidin-alkaline phosphatase and ImmPACT Vector Red Substrate (Vector Laboratories). Sections were counterstained with hematoxylin. 
Immunofluorescent staining. Immunofluorescent staining was performed on frozen sections using the double indirect immunofluorescence method. Frozen sections were incubated with primary antibodies following methanol fixation and blocking procedures as described above. Slides were incubated simultaneously with either anti-ULBP2 $(1: 2,000)$ or anti-ULBP3 $(1: 2,000)$ and mouse $\alpha$-SMA monoclonal antibody (1:500). Primary antibodies were incubated overnight at $4{ }^{\circ} \mathrm{C}$ and signal detected the following day with goat anti-rabbit AlexaFluor 488 and goat anti-mouse AlexaFluor 568 (Thermo Fisher Scientific; 1:500) secondary antibodies. Nuclear counterstaining with DAPI (Sigma-Aldrich; 1:2,000) facilitated histologic identification of tissue orientation. Images were captured using a Nikon A1 Inverted confocal microscope (Nikon) at Cincinnati Children's Hospital Medical Center Confocal Imaging Core. All immunostaining procedures were performed with appropriate negative controls, including: (a) the omission of the primary antibody from the staining procedure, (b) the replacement of the primary antibody with normal rabbit or mouse IgG where appropriate, and (c) the staining of known negative and known positive tissues or cell lines.

ELISA. ELISA kits for ULBP2, ULBP3, and MICA were obtained from Bio-Techne. ELISAs were performed according to manufacturer's protocols. Serum was diluted 1:4 or 1:8, depending on the available volume of sample.

Flow cytometry. NK cell surface staining was performed on thawed PBMC. Antibodies for flow cytometry that were obtained from BD Biosciences included: BV786 mouse anti-human CD3 (clone SK7); BUV395 mouse anti-human CD56 (clone NCAM16.2); BUV737 mouse anti-human CD16 (clone 3G8); R-PE mouse anti-human NKp44/CD336 (clone p44-8); BV510 mouse anti-human NKp46/CD335 (clone 9E2/NKp46); and BV650 mouse anti-human NKG2D/CD314 (clone 1D11). Antibodies obtained from Bio-Techne included: FITC mouse anti-human NKG2C/CD159c (clone 134591). Briefly, cells were quickly thawed, washed in $37^{\circ} \mathrm{C}$ media (RPMI $1640,10 \% \mathrm{FBS}$, phenol redfree) and fixed for 1 hour at RT in 1.6\% paraformaldehyde (Electron Microscopy Sciences). Cells were then washed $2 \times$ in flow buffer $(\mathrm{FB}, 1 \times \mathrm{PBS}, 0.1 \%$ sodium azide, $0.5 \% \mathrm{BSA}, \mathrm{pH} 7.4)$ and blocked with anti-CD16/32 cocktail (eBioscience) and mouse $\gamma$-globulins (Sigma-Aldrich) for 1 hour at RT. Cells were then stained with appropriate antibodies for 1 hour at RT in the dark, washed $2 \times$ with FB, and immediately analyzed by flow cytometry (BD Biosciences). Flow cytometry was performed at Shriners Hospitals for Children's Cincinnati Flow Cytometry Core, Cincinnati, Ohio, USA, using a 5-laser BD LSRII equipped with $355 \mathrm{~nm}$ (UV), $405 \mathrm{~nm}$ (violet), $488 \mathrm{~nm}$ (blue), $553 \mathrm{~nm}$ (yellow/green), and 640 $\mathrm{nm}$ (red) lasers that allow for the detection of 2 scatter parameters and up to 18 fluorescence parameters. Data were collected with Diva 6.02 and analyzed with FCS Express V5 (De Novo Software). Cells were gated first on the lymphocyte population based on forward scatter area (FSC-A) versus side scatter area (SSC-A). Next, doublets were excluded by gating (FSC-A vs. forward scatter height [FSC-H]) on single cells. CD3- cells were then gated, and NK cells were discriminated based on CD56 ${ }^{\mathrm{dim}} \mathrm{CD} 16^{++}$ vs. CD56 $6^{\text {bright }} \mathrm{CD} 16^{-}$. Each NK population then had NKG2D expression examined. Appropriate negative controls were used to set gates for the above populations. UltraComp eBeads (eBioscience) were used to determine the compensation matrix.

$F E V_{1}$ decline. LAM patients enrolled in the NHLBI registry were evaluated at 1 of 6 national centers at 6- to 12-month intervals (20). Due to the irregular timing of some spirometry measurements, intraindividual $\mathrm{FEV}_{1}$ values were averaged from visits that occurred from 24-36 months (30 months) and 42-54 months (48 months) after enrollment. The rate of decline in $\mathrm{FEV}_{1}$ was calculated as the difference between baseline and 30 or 48 months.

Statistics. A 2-tailed Students $t$ test was used for flow cytometry analyses to compare differences between controls and LAM patients. Differences in the rate of $\mathrm{FEV}_{1}$ decline between groups with differing serum levels of NKG2DL was compared by 2-way ANOVA with specific post-hoc contrasts. Systat (v13.1) or R (v3.2.2) programs were used for all analyses, and differences between groups were considered statistically significant when $P<0.05$.

Study approval. Deidentified serum and tissue samples were from the 5-year NHLBI LAM Registry (20) database and repository, curated by the NDRI (Philadelphia, Pennsylvania, USA). Fresh peripheral blood collection was performed at the University of Cincinnati Pulmonary LAM Clinic. Blood was collected after obtaining informed consent. The sample collection was reviewed and approved by the University of Cincinnati IRB (IRB 2013-8157) 


\section{Author contributions}

ARO, FXM, and MTB wrote the manuscript. ARO, RLN, BZY, RF, WRFD, MAN, and HL performed the experiments. JM conducted the NHLBI LAM sample collection. KAWB performed histological analysis and provided pathological descriptions and interpretations. NG provided patient data. ARO performed data analysis. FXM and NG provided clinical samples.

\section{Acknowledgments}

This work was supported by NIH Research grant R01HL119538 and VA-ORD award BX002347 (to MTB). We would also like to thank the LAM foundation for their support.

Address correspondence to: Michael Borchers, University of Cincinnati College of Medicine, Division of Pulmonary, Critical Care and Sleep Medicine, Department of Internal Medicine, PO Box 45267-0564, Cincinnati, Ohio 45267, USA. Phone: 513.558.1027; E-mail: BORCHEMT@ucmail.uc.edu.

1. Carsillo T, Astrinidis A, Henske EP. Mutations in the tuberous sclerosis complex gene TSC2 are a cause of sporadic pulmonary lymphangioleiomyomatosis. Proc Natl Acad Sci U S A. 2000;97(11):6085-6090.

2. McCormack FX, Travis WD, Colby TV, Henske EP, Moss J. Lymphangioleiomyomatosis: calling it what it is: a low-grade, destructive, metastasizing neoplasm. Am J Respir Crit Care Med. 2012;186(12):1210-1212.

3. Vesely MD, Kershaw MH, Schreiber RD, Smyth MJ. Natural innate and adaptive immunity to cancer. Annu Rev Immunol. 2011;29:235-271.

4. Raulet DH. Roles of the NKG2D immunoreceptor and its ligands. Nat Rev Immunol. 2003;3(10):781-790.

5. Cerwenka A, et al. Retinoic acid early inducible genes define a ligand family for the activating NKG2D receptor in mice. Immunity. 2000;12(6):721-727.

6. Bauer S, et al. Activation of NK cells and T cells by NKG2D, a receptor for stress-inducible MICA. Science. 1999;285(5428):727-729.

7. Cosman D, et al. ULBPs, novel MHC class I-related molecules, bind to CMV glycoprotein UL16 and stimulate NK cytotoxicity through the NKG2D receptor. Immunity. 2001;14(2):123-133.

8. Diefenbach A, Jamieson AM, Liu SD, Shastri N, Raulet DH. Ligands for the murine NKG2D receptor: expression by tumor cells and activation of NK cells and macrophages. Nat Immunol. 2000;1(2):119-126.

9. Hayakawa Y, et al. Cutting edge: tumor rejection mediated by NKG2D receptor-ligand interaction is dependent upon perforin. $J$ Immunol. 2002;169(10):5377-5381.

10. Smyth MJ, et al. NKG2D recognition and perforin effector function mediate effective cytokine immunotherapy of cancer. $J$ Exp Med. 2004;200(10):1325-1335.

11. Groh V, Rhinehart R, Randolph-Habecker J, Topp MS, Riddell SR, Spies T. Costimulation of CD8alphabeta T cells by NKG2D via engagement by MIC induced on virus-infected cells. Nat Immunol. 2001;2(3):255-260.

12. Sutherland CL, Chalupny NJ, Schooley K, VandenBos T, Kubin M, Cosman D. UL16-binding proteins, novel MHC class I-related proteins, bind to NKG2D and activate multiple signaling pathways in primary NK cells. J Immunol. 2002;168(2):671-679.

13. Kubin M, et al. ULBP1, 2, 3: novel MHC class I-related molecules that bind to human cytomegalovirus glycoprotein UL16, activate NK cells. Eur J Immunol. 2001;31(5):1428-1437.

14. Chalupny NJ, Sutherland CL, Lawrence WA, Rein-Weston A, Cosman D. ULBP4 is a novel ligand for human NKG2D. Biochem Biophys Res Commun. 2003;305(1):129-135.

15. Bacon L, Eagle RA, Meyer M, Easom N, Young NT, Trowsdale J. Two human ULBP/RAET1 molecules with transmembrane regions are ligands for NKG2D. J Immunol. 2004;173(2):1078-1084.

16. Marcus A, et al. Recognition of tumors by the innate immune system and natural killer cells. Adv Immunol. 2014;122:91-128.

17. Raulet DH, Gasser S, Gowen BG, Deng W, Jung H. Regulation of ligands for the NKG2D activating receptor. Annu Rev Immunol. 2013;31:413-441.

18. Chang YT, et al. Secretome-based identification of ULBP2 as a novel serum marker for pancreatic cancer detection. PLoS One. 2011;6(5):e20029.

19. Paschen A, et al. Differential clinical significance of individual NKG2D ligands in melanoma: soluble ULBP2 as an indicator of poor prognosis superior to S100B. Clin Cancer Res. 2009;15(16):5208-5215.

20. Ryu JH, et al. The NHLBI lymphangioleiomyomatosis registry: characteristics of 230 patients at enrollment. Am J Respir Crit Care Med. 2006;173(1):105-111.

21. Seyama K, Kumasaka T, Kurihara M, Mitani K, Sato T. Lymphangioleiomyomatosis: a disease involving the lymphatic system. Lymphat Res Biol. 2010;8(1):21-31.

22. Smyth MJ, Swann J, Cretney E, Zerafa N, Yokoyama WM, Hayakawa Y. NKG2D function protects the host from tumor initiation. J Exp Med. 2005;202(5):583-588.

23. Hilpert J, et al. Comprehensive analysis of NKG2D ligand expression and release in leukemia: implications for NKG2D-mediated NK cell responses. J Immunol. 2012;189(3):1360-1371.

24. Waldhauer I, Steinle A. Proteolytic release of soluble UL16-binding protein 2 from tumor cells. Cancer Res. 2006;66(5):2520-2526.

25. Wang R, Sun PD. Natural killer cell-mediated shedding of ULBP2. PLoS One. 2014;9(3):e91133.

26. Pappworth IY, Wang EC, Rowe M. The switch from latent to productive infection in epstein-barr virus-infected B cells is associated with sensitization to NK cell killing. J Virol. 2007;81(2):474-482.

27. Richard J, Sindhu S, Pham TN, Belzile JP, Cohen EA. HIV-1 Vpr up-regulates expression of ligands for the activating NKG2D 
receptor and promotes NK cell-mediated killing. Blood. 2010;115(7):1354-1363.

28. Meraj R, Wikenheiser-Brokamp KA, Young LR, McCormack FX. Lymphangioleiomyomatosis: new concepts in pathogenesis, diagnosis, and treatment. Semin Respir Crit Care Med. 2012;33(5):486-497.

29. Lesma E, Chiaramonte E, Ancona S, Orpianesi E, Di Giulio AM, Gorio A. Anti-EGFR antibody reduces lung nodules by inhibition of EGFR-pathway in a model of lymphangioleiomyomatosis. Biomed Res Int. 2015;2015:315240.

30. Lesma E, Grande V, Ancona S, Carelli S, Di Giulio AM, Gorio A. Anti-EGFR antibody efficiently and specifically inhibits human TSC2-/- smooth muscle cell proliferation. Possible treatment options for TSC and LAM. PLoS One. 2008;3(10):e3558.

31. Borchers MT, Harris NL, Wesselkamper SC, Vitucci M, Cosman D. NKG2D ligands are expressed on stressed human airway epithelial cells. Am J Physiol Lung Cell Mol Physiol. 2006;291(2):L222-L231.

32. Vantourout $P$, et al. Immunological visibility: posttranscriptional regulation of human NKG2D ligands by the EGF receptor pathway. Sci Transl Med. 2014;6(231):231ra49.

33. Wu X, Tao Y, Hou J, Meng X, Shi J. Valproic acid upregulates NKG2D ligand expression through an ERK-dependent mechanism and potentially enhances NK cell-mediated lysis of myeloma. Neoplasia. 2012;14(12):1178-1189.

34. Ogbomo H, Michaelis M, Klassert D, Doerr HW, Cinatl J. Resistance to cytarabine induces the up-regulation of NKG2D ligands and enhances natural killer cell lysis of leukemic cells. Neoplasia. 2008;10(12):1402-1410.

35. Liu XV, Ho SS, Tan JJ, Kamran N, Gasser S. Ras activation induces expression of Raet1 family NK receptor ligands. J Immunol. 2012;189(4):1826-1834.

36. Matusali G, et al. Soluble ligands for the NKG2D receptor are released during HIV-1 infection and impair NKG2D expression and cytotoxicity of NK cells. FASEB J. 2013;27(6):2440-2450.

37. Song H, Kim J, Cosman D, Choi I. Soluble ULBP suppresses natural killer cell activity via down-regulating NKG2D expression. Cell Immunol. 2006;239(1):22-30.

38. Whiteside TL. Immune modulation of T-cell and NK (natural killer) cell activities by TEXs (tumour-derived exosomes). Biochem Soc Trans. 2013;41(1):245-251.

39. Viaud S, et al. Dendritic cell-derived exosomes promote natural killer cell activation and proliferation: a role for NKG2D ligands and IL-15Ralpha. PLoS One. 2009;4(3):e4942.

40. Matsui K, Takeda K, Yu ZX, Travis WD, Moss J, Ferrans VJ. Role for activation of matrix metalloproteinases in the pathogenesis of pulmonary lymphangioleiomyomatosis. Arch Pathol Lab Med. 2000;124(2):267-275.

41. Edwards ST, et al. c-Kit immunophenotyping and metalloproteinase expression profiles of mast cells in interstitial lung diseases J Pathol. 2005;206(3):279-290.

42. Ikeda Y, et al. Erythropoietin-driven proliferation of cells with mutations in the tumor suppressor gene TSC2. Am J Physiol Lung Cell Mol Physiol. 2011;300(1):L64-L72.

43. Taveira-DaSilva AM, Pacheco-Rodriguez G, Moss J. The natural history of lymphangioleiomyomatosis: markers of severity, rate of progression and prognosis. Lymphat Res Biol. 2010;8(1):9-19.

44. Wolter F, et al. Hypoxia impairs anti-viral activity of natural killer (NK) cells but has little effect on anti-fibrotic NK cell functions in hepatitis C virus infection. J Hepatol. 2015;63(6):1334-1344.

45. Balsamo M, et al. Hypoxia downregulates the expression of activating receptors involved in NK-cell-mediated target cell killing without affecting ADCC. Eur J Immunol. 2013;43(10):2756-2764.

46. Sarkar S, et al. Hypoxia induced impairment of NK cell cytotoxicity against multiple myeloma can be overcome by IL-2 activation of the NK cells. PLoS One. 2013;8(5):e64835.

47. Dasgupta S, Bhattacharya-Chatterjee M, O'Malley BW, Chatterjee SK. Inhibition of NK cell activity through TGF-beta 1 by down-regulation of NKG2D in a murine model of head and neck cancer. J Immunol. 2005;175(8):5541-5550.

48. Lee JC, Lee KM, Kim DW, Heo DS. Elevated TGF-beta1 secretion and down-modulation of NKG2D underlies impaired NK cytotoxicity in cancer patients. J Immunol. 2004;172(12):7335-7340.

49. Evans SE, Colby TV, Ryu JH, Limper AH. Transforming growth factor-beta 1 and extracellular matrix-associated fibronectin expression in pulmonary lymphangioleiomyomatosis. Chest. 2004;125(3):1063-1070.

50. Nandagopal N, Ali AK, Komal AK, Lee SH. The Critical Role of IL-15-PI3K-mTOR Pathway in Natural Killer Cell Effector Functions. Front Immunol. 2014;5:187.

51. Paquette B, et al. Oestrogen metabolism in lymphangioleiomyomatosis: catechol-O-methyltransferase pathway is not involved Thorax. 2000;55(7):574-578

52. Peraldi MN, et al. Oxidative stress mediates a reduced expression of the activating receptor NKG2D in NK cells from end-stage renal disease patients. J Immunol. 2009;182(3):1696-1705.

53. Antoun A, Jobson S, Cook M, O'Callaghan CA, Moss P, Briggs DC. Single nucleotide polymorphism analysis of the NKG2D ligand cluster on the long arm of chromosome 6: Extensive polymorphisms and evidence of diversity between human populations. Hum Immunol. 2010;71(6):610-620.

54. Tamaki S, et al. Relationship between soluble MICA and the MICA A5.1 homozygous genotype in patients with oral squamous cell carcinoma. Clin Immunol. 2009;130(3):331-337.

55. Aquino-Galvez A, et al. MICA polymorphisms and decreased expression of the MICA receptor NKG2D contribute to idiopathic pulmonary fibrosis susceptibility. Hum Genet. 2009;125(5-6):639-648.

56. Petukhova L, et al. Genome-wide association study in alopecia areata implicates both innate and adaptive immunity. Nature 2010;466(7302):113-117.

57. Romphruk AV, et al. Polymorphisms of NKG2D ligands: diverse RAET1/ULBP genes in northeastern Thais. Immunogenetics. 2009;61(9):611-617.

58. Baragaño Raneros A, Suarez-Álvarez B, López-Larrea C. Secretory pathways generating immunosuppressive NKG2D ligands: New targets for therapeutic intervention. Oncoimmunology. 2014;3:e28497.

59. Spear P, Wu MR, Sentman ML, Sentman CL. NKG2D ligands as therapeutic targets. Cancer Immun. 2013;13:8.

60. Xuan XY, Zhang JF, Hu GM, Li QR, Liu PP, Du Y. Upregulated expression of NKG2D and its ligands give potential therapeutic targets for patients with thymoma. Cancer Gene Ther. 2015;22(7):368-374. 